\title{
MUCOVISCIDOSI: \\ COLONIZZAZIONI BATTERICHE \\ DELLE VIE AEREE INFERIORI ED EVOLUZIONE DELLA MALATTIA.
}

Seca P.; Fabbrizi V.; Sisino L.

Laboratorio Patologia clinica;

Ospedale Civile "G. Mazzini" Teramo - Settore Microbiologia

Introduzione: La Mucoviscidosi è una malattia genetica multisistemica. Il quadro clinico comprende ipersalinità del sudore, insufficienza pancreatica esocrina, grave e progressiva broncopneumopatia cronica. In questo studio abbiamo considerato il decadimento della funzionalità polmonare dei pazienti correlandola con le colonizzazioni batteriche e la fascia d'età, al tipo di mutazione, ai parametri vitali, all'età della diagnosi e alla comparsa di Ps. aeruginosa, B. Cepacia, S. Maltophilia, ritenuti essere i maggiori responsabili del deterioramento delle funzioni polmonari.

Metodi: Lo studio retrospettivo si è protratto dal $1^{\circ}$ gennaio 2001 al 30 aprile 2003; Il follow-up è stato condotto su 55 pazienti, la coorte di soggetti affetti da F.C. afferente presso il "Centro Regionale di controllo per la F.C." del P. O. di Teramo. I campioni provenienti dalle basse vie aeree sono stati processati secondo protocollo, l'identificazione e l'antibiogramma effettuata con il sistema Microscan (Dade Behring) approvato NCCLS.

Risultati: La frequenza della mutazione DF 508 nella nostra coorte è del $34 \%$; non c'è correlazione diretta tra genotipo ed espressione della malattia a livello polmonare. La colonizzazione da Ps.aeruginosa raggiunge il $40 \%$ in valore assoluto in pazienti superiori a 30 anni. Nessun paziente con colonizzazioni croniche da Ps. aeruginosa mostra una fev1 superiore all'85\%; parametri vitali critici si osservano in pazienti con colonizzazioni croniche da B. cepacia e S: maltophilia. Osserviamo parametri vitali compromessi anche in soggetti mai colonizzati da Pseudomonas sp..

Conclusioni: Le colonizzazioni croniche da Pseudomonas sp e la degenerazione dei parametri vitali non sono sempre in correlazione diretta. Più correlabile ai parametri vitali è la risposta infiammatoria a queste colonizzazioni, come affermato da varie scuole di pensiero e dall'uso dei macrolidi nelle esacerbazioni della malattia polmonare. Nella degenerazione polmonare nella mucoviscidosi riteniamo quindi punto focale la diversa ed incostante espressione fenotipica di un'alterazione della risposta immunitaria specifica e aspecifica. 